\title{
Smart Stadium using Cloud Computing and Internet of Things (loT): Existing and New Models
}

\author{
Mustafa Jamal Mahdi \\ College of Engineering \\ Al-Iraqia University \\ Baghdad, Iraq
}

\author{
Abbas Fadhil Aljuboori \\ College of Engineering \\ University of Information \\ Technology and Communications \\ Baghdad, Iraq
}

\author{
Mudhafar Hussein Ali \\ College of Engineering \\ Al-Iraqia University \\ Baghdad, Iraq
}

\begin{abstract}
In our life technology is important there are two entirely various technologies are cloud computing and the IoT and both are very portion of our lives. They are supposed to be more common in their acceptance and use, making them essential ingredients of the future Internet worldwide. Because of the lack of time in our working life and the follow-up of all operations that we must follow before any match is held on any stadium in the world. An aspect of precautionary measures is discussed here before every match. In this research, a discussion was conducted on how to integrate cloud computing and the IoT and use them to work in developing stadiums in the word and made it smart. Several existing and new models of smart stadium are although explained.
\end{abstract}

Keywords: IoT, Cloud Computing, Smart Stadium, Web Application, Smart Application.

\section{INTRODUCTION}

Smart Stadium for Smart Living is an effort intended to integrate IoT and smart stadium technology for organizations and partners. The initiative joins Arizona State University in Tempe- Arizona: Dublin City University in Dublin- Ireland: Gaelic Athletic Association of Ireland; and Intel Corporation to turn two stadia ASUs Sun Devil Stadium and Ireland's Croke Park Stadium into twinned smart stadia with the possibility to be world kind testbeds for exploring smart stadium apps and IoT solutions. To date, these initiatives have concentrated on two wide areas of implementation. 1Enriching the fan/attendee trial, and 2- Improving operations at the stadium. While the implementation focus of these initiatives is set in the framework of events related to stadiums, they are applicable to broader areas of application for smart cities. Under these projects, the complete spectrum of projects covers crowd control, fan participation, event planning, by ply use a set of sensors like video cameras and mounted microphones, stadium security and environmental for monitoring issues [1]. Smart Stadium is an increasingly popular fact in cities across the world. The concept of a smart stadium is not recent, but it has changed largely due to the advent of new technologies, while implemented, allows smarter resources and processes to extend the stadium's ability to function in a more effective, scalable, interactive, and sustainable manner [1]. Football is one of the world's most common sports, which is why all suitable conditions for growth and advancement must be given. And in terms of setting rules, designing, and improving this game in all respects, the party responsible for this is FIFA. Many advancements that have changed the game have recently been implemented by the company responsible for the growth and advancement of the standard of football worldwide. For instance, Goal Line Technology (GLT) has been implemented to decide if the entire ball has reached the goal line to help referees make their decisions. [2][3]. Furthermore, to build safe and comfortable environments, FIFA has issued technical guidelines and criteria for the construction of new football stadiums [4]. Of requirements and things organized a special football pitch should note that several aspects including lighting. for control and track the function of lighting towers, it relies on IoT and cloud computing. It represents one of the most disruptive innovations, allowing computing scenarios that are omnipresent. Internet of thing is described by tiny, widely distributed real-world items with fixed storage and processing ability that involve issues of accuracy, efficiency, safety, and particularity [3]. It features a smart development of the playground that supports IoT to evaluate application requirements and recommend architecture for the cloud of things. This is achieved by incorporating the connections with the surrounding world between new-generation services, where the following is centered here: 1- Designing a piece of common evidence to merge the cloud with the IoT. 2Developing and testing the application based on the IoT and the cloud. 3- Developing devices for this work to facilitate interactions with smart devices using the cloud. 4- Improving in the mechanism of work and making the exchange of dataefficient using JavaScript [6]. The Internet of Things is a term rich with many specifics and modern technologies that, luckily, are set to change life dramatically in our modern world. This means that the IoT is a wide community of devices that are interconnected used to collect and exchange information between devices and to store, evaluate, or analyze the effects of that information [7]. Stadium operators are in a squeeze game between customers expecting a more immersive experience and host stadium seeking higher returns on the dynamic investments of their sport. They need to find new ways to fill the arena, push fans to spend more, and keep everyone healthy with today's advanced home entertainment systems and the latest stats and alerts available on mobile devices [3].

\section{CLOUD COMPUTING}

Over the past few years, cloud computing has emerged as a transformative model with the potential to convert IT companies and make them more flexible and lither than no prior. Cloud computing can especially allow complex organizations such as stadiums to operate more effectively, create new opportunities, and open new business models because of its numerous and important advantages. The debate about how cloud computing can enable stadiums to become smart has revealed a multitude of applications, in any way of the size and level of organization and resources of a 
community, albeit obviously at an early stage. [4]. Economic advantages, pace, nimbleness, versatility, high elasticity, and more creativity are promised by Cloud Computing. The organizations reasons for migrating their apps to the Cloud are nearly connected to the next main features of Cloud [4]. There are five important countenances of cloud computing listed below, by depended on the report of the National Institute of Standard Technology. [5]:

- Broad Network Access. Cloud computing services are ready and deliver over the network and are utilized through much client apps for various types of platforms such as PDAs and mobile phones. Resource Pooling. The services of the provider are gathered to be used by various clients using a form of multi-renter with various resource allocated and assigned dynamically according to the order of the client.

- Cloud computing elasticity has an infinite number of services; at any time and quantity, these resources can be delivered from provider to customer. As the application load increases and vice versa, the given resource can be automatically increased.

- $\quad$ Measured Service Although several different clients (such as multi-tenancy) share and pool computing resources, the cloud infrastructure can use appropriate mechanisms to calculate what resources have been used by each individual client. The hiring rate varies from one cloud provider to another.

\subsection{Cloud Computing Ingredients}

Cloud computing have three basic Ingredients as follows [6]:

- Client Computers: Using client computers, the enduser may communicate with the cloud.

- Distributed servers: they are servers spread between various locations but behave as though they were working together.

- Data Centers: The compilation of servers are the data centers.

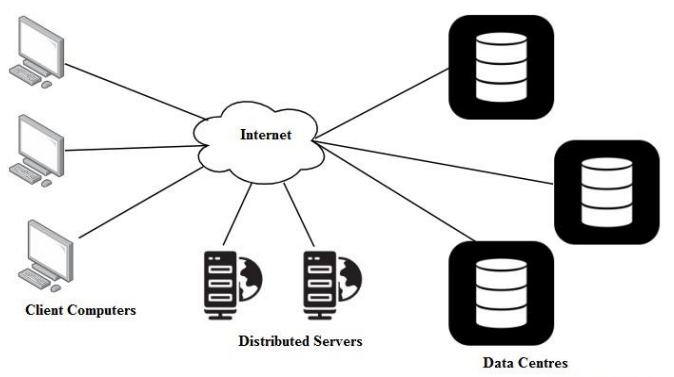

Figure 1. Ingredients of Cloud Computing

\subsection{Cloud Computing Services}

The cloud infrastructure can be split into the front end back end the and. The front end is made available to the user over Internet connection, allowing the system to communicate with the user. [7]. The back end includes the different models of cloud services:

- $\quad$ Software as a Service. (SaaS)

The user is given a hosted collection of software working on a platform and infrastructure owned by the cloud provider. Apps are planned and built to be accessed over the Internet by different cloud customers at the same time. CSP, which maintains and ensures the up to date working of the system, manages the hosted program. Multitenancy is provided by the hosted framework, is ready onrequest, and can be scaled up to down. Some SaaS providers operate on PaaS or IaaS offerings from other cloud providers. [7]. Example of SaaS: Email and Office output: Email apps, word editors and processors, spreadsheets apps, presentations apps are model examples in this denomination. [8].

- $\quad$ Platform as a Service (PaaS)

$\mathrm{PaaS}$ is a development service that is provided to users through the Internet. No software installation or hardware specifications are required by the user, thereby saving costs. It is a middleware that has built-in tools, built-in protection, and web service interfaces for the deployed applications on which PaaS applications are built. You can integrate the deployed application on the same platform with other applications and interface it with else apps outside of the platform. "PaaS" has software combine a database, middleware, and evolution material.[7]. proverb of "PaaS" [8]:

a) Application Deployment.

b) Database.

c) Development and Testing.

d) Business Intelligence.

e) Integration.

- Infrastructure as a Service. (IaaS)

These is the distribution of servers as a service, storag, network, and operating system. IaaS requires an imaginary computer that the operating system has formerly installed and formation. IaaS enable the storage of data in different geographical location. Providers monitor cloud data center operations while enabling users to implement and manage computing services themselves with flexibility. The user has arrival to virtual machines, storag, network infrastructure, device deployment, and running computing resources. The cloud provider handles only SW and HW, such as servers, host OS, storage devices and virtualization hypervisors. A standard graph of the cloud architecture available to cloud users [7]. Ex. of I-aa-S [8]:

a) Content Delivery Networks: To enhance system rendering, such as velocity and cost linked with the acceptance of content for web-based applications, CDNs recording user content and files. This is helpful in handling various types of content for any websites or mobile apps.

b) Backup and Recovery: This offers the capability to backup and restores files seamlessly.

c) Compute: This requires server specifications to manage cloud services that can be dynamically purvey and configured. 
d) Storage: Storage that is extremely scalable capacity is also available for recording application activities, file backups, and file recovery and storage.

\section{INTERNET OF THINGS (IOT)}

Smart and self-formation nodes connected in complex and universal network infrastructure are the foundation of the IoT paradigm. It is one of the most innovative inventions for universal and popular computing scenarios.

Internet of Things is the true world and tiny items with restricted storage and processing ability and consecutive issues of fineness, efficiency, protection, and singularity are generally [9]. The IoT is a network that, according to specified agreements, via RFI, infrared sensor, universal position systems, laser scanner, and other datum-sensing devices, it intelligently recognizes, locate, track, monitor, and manages objects. [10]. The awareness layer, the network layer, and the apps layer are included in the basic Internet of things architecture; see Figure 2 [11].

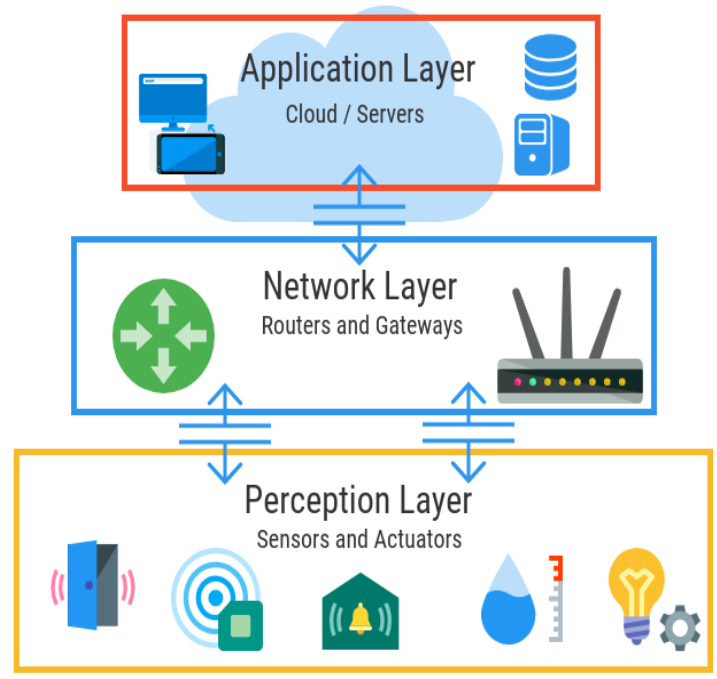

Figure 2. The Basic Architecture of IoT

- Perception layer: To fix the issue of data collecting in the material world and to realize the target of detailed data perception, the perception layer first Collecting data from the outer material environment over cameras, sensors, and other devices. The principal technologies of the perception layer include (RFID), sensor networks, and so on.[11],[12].

- Network layer: Data obtained in the network layer is distributed over current Internet, the connection web, the radio and tv networks, the different arrival networks, and special networks. The transport layer's core technologies are far- reaching wire and wireless connection protocols, network incorporation technology, and intelligent mass information processing technology.[11].

- $\quad$ Application layer: To obtain a wide variety of smart apps solutions, like Smart Stadium and smart transmission, the apps layer combines IoT technology with real industry apps. [13], [11]. see Figure 3. Intelligent computing technologies like edge computing, cloud computing, machine learning, and so on are the main technologies in the application layer.

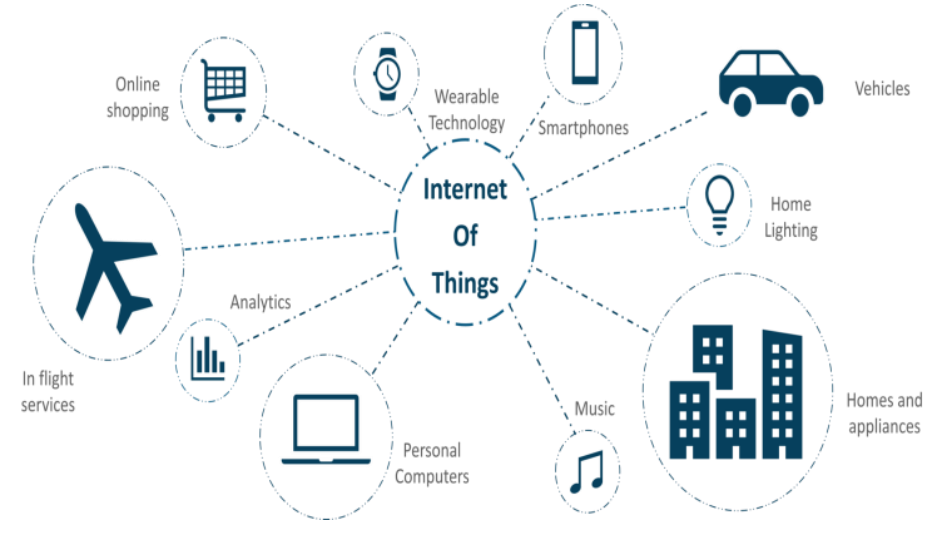

Figure .3 Different Apps of IoT in Human Life

\subsection{Technologies of IoT}

Different IoT classification technologies are used, but the four prime techniques are as follows: [14]:

a) Radio Frequency Identification "RFID".

b) Machine to Machine Communication "MtoM".

c) Vehicle to Vehicle Communication "VtoV".

d) Near Field Communication "NFC".

\section{a) Radio Frequency Identification "RFID”.}

RFID itis a system consisting of two components: a datacontaining wafer and a reading interface. The number of individuals assessed is dependent on the method of granting entry to the building. A simple estimate of space use can be provided by calculating the number of check-ins [15]. RFID technology is based on readers and tags, as we have already mentioned, so RFID describes three configurations in the initial research stage.[14]: -

- $\quad$ active RFID

- $\quad$ passive RFID

- $\quad$ active Reader Active Tag.

b) Machine to Machine Communication

M2M The referred is to connection among computers, embedded processors, smart sensors, actuators, and mobile devices. In this case, the use of M2M connection is increasing at a quick rate. For example, researchers estimated that, except for mobile phones, yon will be 1.5 billion wireless linked devices by 2014. Currently, there are almost. 2 billion wirelessly linked devices that can collect information from the sensors, analyze that information, and send the information to other devices to implement some mission. The device receives the information and executes task with the assistance of triggers, sensors, embedded processors, and apps software.[14].

\section{c) Vehicle to Vehicle Communication}

In that technique, the objects are cars, that can connect with other car or the sensors around there. The key point of worry here is that, since the object move and interacts with else movable object or for the sensors at the wayside, there is no proper method of specifying the protocols. So, we are unable 
to identify any protocol for routing. This interaction will function for a long distance and create successful contact between objects'. This technology was mainly developed for the purposes of transit control, protection, and prevention of accidents. [14].

\section{d) Near Field Communication (NFC)}

NFC is it integrates an RFID reader into a mobile phone, somehow a bit like RFID, which makes it faster, more secure, and more effective for users. NFC is a low-extent wireless technology for a hesitation of $13.56 \mathrm{MHz}$, work for so short distances up to $4 \mathrm{~cm}$. Complementing Bluetooth and 802.11 with the tall-extent capabilities at a range of above at $10 \mathrm{~cm}$, NFC allows for conjectural initialization of wireless networks. It was first founded by companies such as Philips and Sony. The exchange of data was approx. $424 \mathrm{kbps}$. In NFC, during data reading, energy exhaustion is beneath 15 ma.In NFC technology, there are two modes [14]: Active. Passive.

\section{CLOUD AND IOT: THE NEED FOR THEIR INTEGRATION}

The cloud world and IoT world have visible a fast and separate development. Both world are so unlike from any other and, until best, they features are also complement, as Table 1 implies. The main explanation of why much researchers have propose and are proposing their integral is such complementarity, usually to gain benefits in specific applications [9].

Table 1. Aspects of Cloud and IoT

\begin{tabular}{|c|c|c|}
\hline & Internet of Things & Cloud Computing \\
\hline Displacement & Pervasive & Centralized \\
\hline Reachability & Limited & Ubiquitous \\
\hline Components & Real word "things" & Virtual \\
\hline $\begin{array}{c}\text { Computation } \\
\text { al capabilities }\end{array}$ & Limited & Virtually \\
\hline $\begin{array}{c}\text { Role of the } \\
\text { Internet }\end{array}$ & $\begin{array}{c}\text { Point of } \\
\text { convergence }\end{array}$ & $\begin{array}{c}\text { Means for delivering } \\
\text { services }\end{array}$ \\
\hline Big data & Source & Means to manage \\
\hline
\end{tabular}

In general, Internet of things can take feature of the Cloud's nearly infinite capacities and resources to compensate for its technical shortcomings for instance storage, processing, communication. Cloud may provide an efficient sol for IoT operation, department, and installation as well as for execution apps and services that take advantage of the stuff or data generated via them. On the different part, the Cloud would interest of IoT via expansion itis range to deal with real world things in a extra distributed and dynamic way, and to offer new services in a wide variety from real life scenarios.

In Tab.2, the finished Cloud and IoT feature emerging from the various literature proposals and exciting the Cloud IoT paradigm are reported.

Table 2. Complementarity and Integration of Cloud and IoT

\begin{tabular}{|l|l|}
\hline IoT & Cloud \\
\hline
\end{tabular}

\begin{tabular}{|c|l|}
\hline $\begin{array}{c}\text { Pervasive (things placed } \\
\text { everywhere) Ubiquitous } \\
\text { (resources usable from } \\
\text { everywhere) }\end{array}$ & $\begin{array}{l}\text { Ubiquitous (resources usable } \\
\text { from everywhere) }\end{array}$ \\
\hline $\begin{array}{c}\text { Real world things Virtual } \\
\text { resources }\end{array}$ & Virtual resources \\
\hline $\begin{array}{c}\text { Limited computational } \\
\text { capabilities Virtually } \\
\text { unlimited computational } \\
\text { capabilities }\end{array}$ & $\begin{array}{l}\text { Virtually } \\
\text { computational capabilities }\end{array}$ \\
\hline $\begin{array}{c}\text { Limited storage or no storage } \\
\text { capabilities Virtually } \\
\text { unlimited storage capabilities }\end{array}$ & $\begin{array}{l}\text { Virtually unlimited storage } \\
\text { capabilities }\end{array}$ \\
\hline $\begin{array}{c}\text { Internet as a point of } \\
\text { convergence Internet for } \\
\text { service delivery }\end{array}$ & Internet for service delivery \\
\hline $\begin{array}{c}\text { Big data source Mean to } \\
\text { manage big data }\end{array}$ & Mean to manage big data \\
\hline
\end{tabular}

- The problems solved and the benefits achieved by implementing the Cloud IoT paradigm are described below [16]:

- $\quad$ Storage resources. IoT offers a broad range of sources of information "like, stuff" with three Big Data features that generate a large amount of nonstructured or semi structured data: volume "like, data size", variety "like, data types", and velocity "like, data generation frequency".

- Computational resources. IoT systems have restricted resources for processing and don't allow data processing on- location. Data composed is typically transmitted to extra efficient nodes where it is possible to aggregate and process, but without proper infrastructure, scalability is difficult to achieve.

- Communication resources. One of the IoT specifications into allow IP_enabled devices to connect via dedicate hardware, and it can be very costly to support such communication. Cloud provides an easy and affordable solution that uses personalized portals and built-in apps to link, monitor, and manage something from anywhere and anytime.

- New capabilities. Very height frequency from devices, technologies, and protocols characterizes the IoT. It can therefore be very hard to obtain scalability, interoperability, reliability, performance, availability, and security.

\section{SMART WEB APPLICATION IN SMART STADIUM}

One of the findings of the case study was that the knowledge produced by the instruments could be better used to monitor the stadium and around it. And optimize the advantages of the tools through this. An application that can be used by Stadium management often offers useful feedback, as seen by the Edge Olympic, for the data lake. These could have a beneficial effect on the declared objectives for each smart device, however on cost reduction and user experience support. This type of approach can easily be implemented on the network if a stadium has a rigid basic infrastructure. [15] 
IoTCloud is an open-source project aim at merge stuff (smartphones, tablets, robots, websites, etc..) with background to handle sensors and they messages and to provide an API for apps that are concerned in these data. The Cloud may provide an efficient solution for implementing the management and composition of IoT resources as well as applications that take advantage of stuff or data generated by them.[9]. CloudIoT has given origin to a new collection of intelligent services and apps so as to can have a huge effect on daily life as shown in. Figure .4[9].

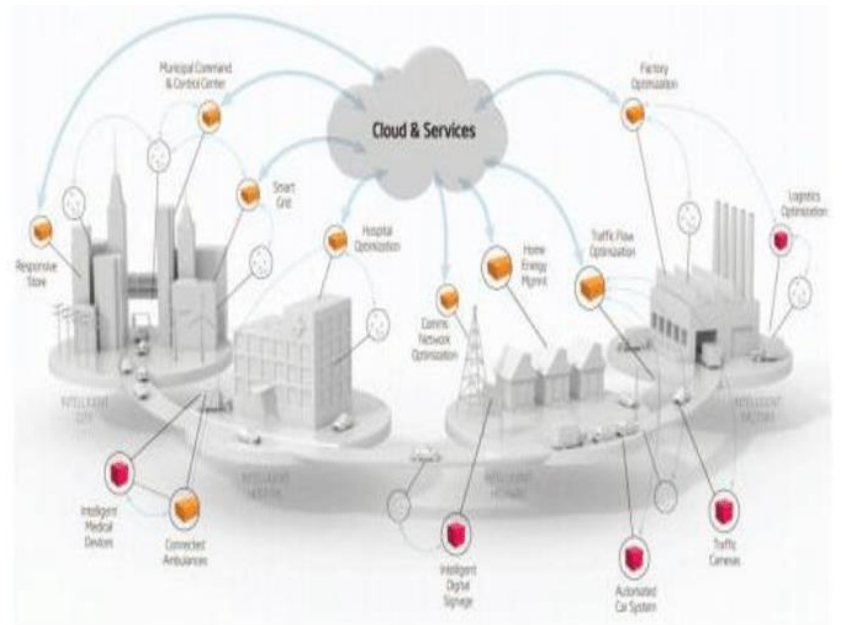

Figure 4. Services made potential thanks to the CloudIoT paradigm

Three layers are used in the standard web application: presentation, application, and database. However, you usually see the presentation layer divided into two separate layers when dealing with stable and modular web applications: client and representation. Figure. 5 shows the three_tier web pattern [17].

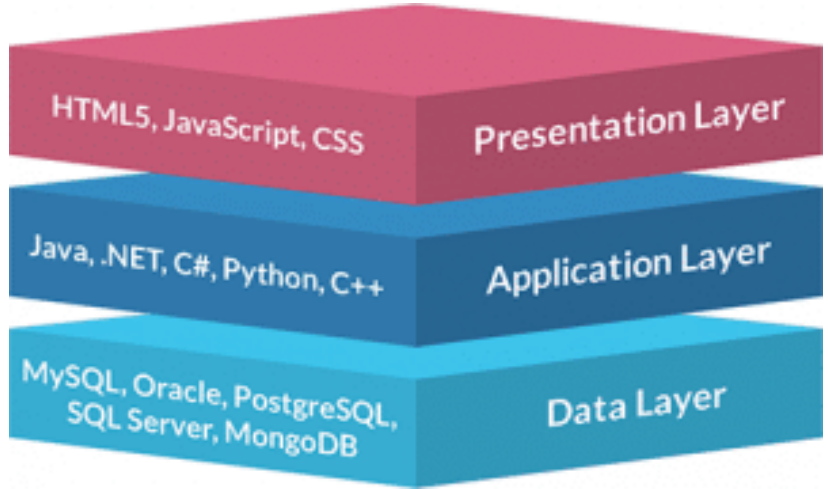

Figure 5. The Three tier_web_pattern

a) The- tier - web pattern

It is crafted to relief developers to read the core components of the framework for developers to make it more flexible and scalable. This architecture is planned to have an overall public-facing interface. By divide an app into many levels, he can install middleware, safeguard every individual layer, and until give up layers to untrustworthy third-party apps. [17]. Figure.6 show an alternate version of the tiered web style that splits the presentation layer to two layers. The developer will offload the client layer to the individual client by breaking the display layer into the client and representative layer to effectively provide several different clients with a single representation.

\section{Client}

\section{Representation}

Application

\section{Database}

Figure 6. The four tier web pattern

b) Reasons for Usage

If any of the above applies, the developer wants to use this pattern: Needed to build a modular cloud system. To have many interfaces for his system. And link different apps to every other (using Amazon cloud services, Google Cloud... etc.). [16].

c) Implementation

A standard implementation of an $\mathrm{n}$ tier architecture is based on a four-tier architecture. The most significant thing is whither each layer goes...Figure.7 illustrates how much a develop can divide the layers that it cloud services offer.

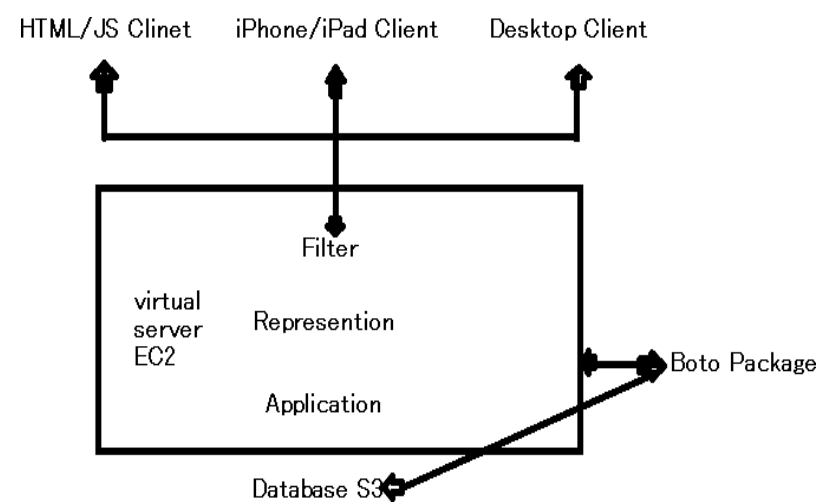

Figure7. Deployment of the n-tier architecture

In the ' $n$ '-tier architecture system, three distinct client layers are given: one for basal HTML and JScript, the other for iPhone clients device and iPad clients device, and a tertiary for desktop clients. Each of this three layers will sitting the user with the data and set it up directly on the client's device. A developer has almost no faith in anything coming from either of these clients, so all authentication and authorization below this layer must be done by a programmer. The candidate layer that allows a developer to recap the authenticate and license from the representation and app layers is straight underneath client layers. It sits direct on top of the layer of representation, altering any input and output flowing in which. Both two layers need to talk same protocol of communication.

\section{LITERATURE SURVEY}

Looking to the smart stadium's institutional context helps us positioning the overarching rationale of innovation crisscrossing the "permeable boundaries" of the stadium, who no way to stands alone in the analysis. We discuss how the stadium is built by the IoT and Cloud Computing techniques 
and university projects, which serve as an information infrastructure that conditions those facts, techniques, and smartness narratives to be applicable in the present.

S. Panchanathan et al [1] The Authors discuss here on three fan enrichment initiatives were presented within the framework of the Smart Stadium for Smarter Living action. These ventures centered on enhanced protection Crowd Under-standing, fan participation Sporty Demonstrator Platform, and deficiency /convenience (Wait Time/Queue Estimation). Each data collection was split into a classified training set and an unclassified set randomly. 10 percent of the dataset size was set to the batch size. From the unclassified set, a batch of samples was queried, appended to the classified collection, and the output was assessed on entire unclassified collection.

M. M. Froufe et al.[2] The Authors discuss here on proposes that the key drivers and systems of smart buildings be identified and compared by associating them with the main beneficiaries: consumers, owners, and the climate. On the basis of the approach adopted, 11 drivers and eight systems have been identified and drivers have been grouped into three groups from the study of the key beneficiaries: consumers primarily concerned with health, wellbeing and meeting expectations; owners primarily concerned with improving the cost benefit ratio; and the environment primarily concerned with reducing consumption and pollution. The analysis had three main objectives: first, to discuss the main drivers that improve the knowledge of a building by rating them according to the main beneficiaries; second, to investigate the main systems that are sitting in those buildings; and third, to investigate the relationship between drivers and systems. We used a three step way to achieve these objectives:

bibliographic scan, identification of the smart building's key drivers and systems, and find of the relationships between drivers and systems.

S. A. Alhadad et al [3]. The Authors focus here on Addresses the importance of the use of specialized equipment in the area of a sports organization. It starts with an introduction of how, with live examples, they are of a paramount benefit to either the fans of the stadium's infrastructure facilities, e.g., Parking lot entrance, the ability of the controlling smart systems to detect any emergency or defect to handle the problem at once. After that, how the information security system work in the stadium, accompanied by exclusive and unique pictures and graphics. Three producers are essential in the first stage, cameras, sensors, and the IoT gateway. Cloud analytics rabidly capture and evaluate them, allowing stadium managers to make data-driven decisions.

C. Kakderi et al [4]. The authors also concentrate on the STORM CLOUDS model as a sol for municipalities everywhere (1) deploying a purse of governance, economic and quality of life smart city applications on a single cloudbased platform, and (2) using the platform and its corresponding resources to move their current appls to the cloud environment. It plays an important role in making efficient use of services that are distributed. Also discussed was the expansion of SCP for the rapid deployment of sensors and motors connected to the network at the city level and three modern modules must be developed to back up the basic reform of IoT solutions. 1- The control unit will deal with the interface of the device and enable the risky response in time as it is composed of the interface and the operating system. 2A data provisioning unit that provides a unified acquisition of sensor data to enable additional processing steps as it will store the data obtained from the SCP data service layer. 3The Big Data Analytics Unit will provide real analytics, identify data of interest, and improve algorithms and services.

M. U. Bokhari et al [5] The authors focus primarily on the concept of cloud computing technology and different levels of cloud architecture. Also, cloud service and implementation models are discussed briefly. Comparison of different cloud computing services models, implementation models: private, public, and community cloud, as well as public and private cloud information security specifications according to different service models, have been provided. Cloud computing, however, has many features, including on-demand self-service, large network connectivity, pooling of resources, elasticity, and calculated service, but it cannot be fully trusted. Finally, the main challenges and issues of cloud computing with regards to security for further research have been discussed in the chapter.

P. Srivastava et al [6]. The authors focus primarily on the brief review of the implementation, development, forms, and components of cloud computing in cloud computing, as well as various cloud computing methods and some of its benefits. The cloud computing application area will be gradually rising. Cloud computing is now used by almost all small and large industries to handle storage, traffic, and hardware requirements.

Odun-Ayo et al. [7]. The key objective of the authors is to define, analyze and clarify the emerging developments and growth in the architecture of cloud computing.

A. Botta, W. De Donato, V. Persico, and A. Pescapé.[9] The Authors focused on how we integrate cloud computing and the Internet of Things, or what we call CloudIoT. And look for important challenges such as hardware and technology inconsistencies, hardware, reliability, scalability, and safety. He also showed that the CloudIoT model is related to energy, energy efficiency, etc.

J. H. Nord et al [10] The authors focused on establishing an extensive review and explanation of the IoT, including discussion of IoT architecture, applications, and impact. In addition, IoT priority areas challenges were identified, and a theoretical framework and conceptual model-based.

G. Mei et al. [11] The authors concentrated on IoT implementations, innovations, and problems in the protection of geo-hazards. The IoT is commonly use in the preventing of three collective classifications of geo-hazards: (1) collapse of slopes, include landslides, flow of debris and Rocks (2) surface deformation, include surface clefts, collapsing of surfaces and decline of surfaces, and (3) Earthquakes. Risks. The emphasis was on providing a detailed survey of applicable IoT research and technical advances related to the prevention of geo-hazards. In the monitoring and early warning of seven types of common geo-hazards, including landslides, debris flow, rockfall, soil subsidence, surface collapse, surface cracks, and earthquakes, it first surveys the IoT implementations, then examines the main geo-hazard mitigation technologies while using the IoT. And the concerns of IoT-based tracking and early warning systems for geohazard avoidance are finally summarized. Furthermore, the possible recommendations for using the IoT for the protection of geo-hazards are also illustrated.

A. Tiwary et al [13] The authors concentrated on IoT and its applications in various science and technology fields. It is also given alongside the launch of the IoT literature review. It also addresses the design and components of the IoT along with its 
various applications. Architecture and various IoT elements are clarified. It also defines the main features and their implementations.

S. van Heck [15] focused on the following research question to be answered in this study: what smart instruments maybe found in stadiums, and how much can this smart tools be configured for use? And address the stadium-focused questions: what smart tools maybe found in stadiums, and how can to be configured to use these smart tools? To maximize the use of the tools, what interventions can be recommended? What progress has been made since the advent of intelligent tools, and what can be improved? What smart tools are implemented at a stadium and what are the targets to their integration? What features do smart stadiums have? What are the features of a stadium and how does the need for smart tools apply to this? How is the integral of smart tools concerning to real ownership management theories? What are smart tools?

M. Puneet et al [17] The authors concentrated on how to develop any cloud web application, provide multiple interfaces for it, and how to select the best Amazon Web Services service for an application. The authors concentrated on how to develop any cloud web application, provide multiple interfaces for it, and how to select the best Amazon Web Services service for an application. And if developers want to use Amazon cloud services and can easily create applications using the cloud, the cost of cloud computing is also small since all the software and hardware available are leased from cloud services for use time and high performance that the application obtained from cloud services.

\section{CONCLUSIONS}

The growth of IoT software is turning our environment into one previously considered impossible. The IoT will service our community by growing efficiency, integrity, and convenience for both businesses and consumers alike. However, Internet of things involves key legal concerns for any new technology, such as protection, privacy, data management, and the need for standards and protocols. If left unchecked, the IoT could have passive effects. If dealt with successfully, however, the IoT will flourish while maintaining individuals' fundamental rights. newly, sensors with high sensibility and low price are on the market. With the evolution of the Internet, we can send and receive data anywhere in the world. In addition, device and storage virtualization and software-defined network development enable the efficient use of ICT for infrastructure in many fields. Technologies and principles in various areas, such as civil engineering and ICT, will be integrated in the near future. In addition, social infrastructures would be virtualized by ICT in the real world. A smart society can then be realized where, by accurate data analysis and control, we can run the infrastructure. The cost of cloud computing is also small since all the software and hardware required is leasehold-for-use time as well as high execution performance that the app acquires from cloud providers, and we can easily develop the application using the cloud.

\section{REFERENCES}

[1] S. Panchanathan et al., "Enriching the fan experience in a smart stadium using internet of things technologies," Int. J. Semant. Comput., vol. 11, no. 2, pp. 1-34, 2017, doi: 10.1142/S1793351X17002751.

[2] M. M. Froufe, C. K. Chinelli, A. L. A. Guedes, A. N. Haddad, A. W. A. Hammad, and C. A. P. Soares, Smart Buildings: Systems and Drivers, vol. 10, no. 9. 2020.

[3] S. A. Alhadad and O. G. Abood, "Enhancing Smart Sport Management based on Information Technology," IOSR J. Sport. Phys. Educ. (IOSR-JSPE, vol. 5, no. 5, pp. 1926, 2018, DOI: 10.9790/6737-05051926.

[4] C. Kakderi, N. Komninos, and P. Tsarchopoulos, "Smart cities and cloud computing: lessons from the STORM CLOUDS experiment," J. Smart Cities, vol. 2, no. 1, 2016, DOI: 10.18063/JSC.2016.01.002.

[5] M. U. Bokhari and Q. Makki, "A Survey on Cloud Computing A Cloud architecture A Security," pp. 149164, 2018.

[6] P. Srivastava and R. Khan, "A Review Paper on Cloud Computing," Int. J. Adv. Res. Comput. Sci. Softw. Eng., vol. 8, no. $6, \quad$ p. $17, \quad 2018$, DOI: 10.23956/ijarcsse.v8i6.711.

[7] I. Odun-Ayo, M. Ananya, F. Agono, and R. GoddyWorlu, "Cloud Computing Architecture: A Critical Analysis," Proc. 2018 18th Int. Conf. Comput. Sci. Its Appl. ICCSA 2018, pp. 1-7, 2018, DOI: 10.1109/ICCSA.2018.8439638.

[8] H. opencrowd. co. Cloud Taxonomy .

[9] A. Botta, W. De Donato, V. Persico, and A. Pescapé, "Integration of Cloud computing and Internet of Things: A survey," Futur. Gener. Comput. Syst., vol. 56, pp. 684-700, 2016, DOI: 10.1016/j.future.2015.09.021.

[10] J. H. Nord, A. Koohang, and J. Paliszkiewicz, "The Internet of Things: Review and theoretical framework," Expert Syst. Appl., vol. 133, pp. 97-108, 2019, DOI: 10.1016/j.eswa.2019.05.014.

[11] G. Mei, N. Xu, J. Qin, B. Wang, and P. Qi, “A Survey of Internet of Things (IoT) for Geohazard Prevention: Applications, Technologies, and Challenges," IEEE Internet Things J., vol. 7, no. 5, pp. 4371-4386, 2020, DOI: 10.1109/JIOT.2019.2952593.

[12] P. P. Ray, "A survey on Internet of Things architectures," J. King Saud Univ. - Comput. Inf. Sci., vol. 30, no. 3, pp. 291-319, 2018, DOI: 10.1016/j.jksuci.2016.10.003.

[13] A. Tiwary, M. Mahato, A. Cheddar, M. K. Chandrol, M. Shrivastava, and M. Tripathi, "Internet of Things (IoT): Research, Architectures, and Applications," Int. J. Futur. Revolut. Comput. Sci. Commun. Eng., vol. ISSN, no. 4, pp. 2454-4248, 2018, [Online]. Available: http://www.ijfrcsce.org.

[14] V. K. B., S. L. Joshi, and S. H. Barshikar, "A Survey on Internet of Things," Int. J. Comput. Sci. Eng., vol. 6, no. 12, pp. 492-496, 2018, DOI: 10.26438/ijcse/v6i12.492496.

[15] S. van Heck, "Smart Stadium Tools," no. June 2019, [Online]. 
International Journal of Computer Applications Technology and Research

Volume 10-Issue 05, 111-118, 2021, ISSN:-2319-8656

Available:https://repository.tudelft.nl/islandora/object/uu id:4e3536e 0-f6c3-458c-8ac0-

ba81b85a5ba3/datastream/OBJ/download.

[16] J. Angelin Jebamalar and A. Sasi Kumar, "A review on the integration of cloud computing and internet of things," Int. J. Eng. Technol., vol. 7, no. 2.33 Special
Issue 33, pp. 683-684, 2018, doi: 10.14419/ijet.v7i2.33.15475.

[17] M. Puneet, J. Kaur, and M. Pallavi, "International Journal of Software and Web Sciences ( IJSWS )," no. October 2016, pp. 54-57, 2013 